\title{
Micromanipulation of cells and particles using ultrasonic fields
}

Martyn Hill, Peter Glynne-Jones, Nicholas R. Harris, and

Rosemary J. Boltryk

Ultrasonic standing waves allow concentration, washing, fractionation, or trapping against a flow of cells in microfluidic environments and can potentially enhance biosensor performance.

Micrometer-scale particles, including cells, can be manipulated using the acoustic radiation forces that are generated by nonlinear interactions between acoustic scattering from the particles and the energy gradients within an ultrasonic field. Such forces can be created by exciting a resonant field within a fluid-filled chamber and, although high-energy ultrasound is well-known for causing cell damage, the energy densities needed to move cells are generally below the levels that reduce cell viability. ${ }^{1}$ The technology is well suited to integration within lab-on-a-chip devices. It is complementary to optical trapping because the potential wells generated are relatively large, thus making ultrasound suitable for the formation and manipulation of cell agglomerates but less suitable for precise manipulation of individual cells.

The magnitude of the radiation force scales with both the volume of the particle and the frequency and energy density of the acoustic field. It is also a function of the 'acoustic contrast factor,' which depends on the relative compressibilities and densities of the particle and its surrounding fluid. ${ }^{2}$ Under such a force, the majority of cells will move towards regions of low acoustic pressure (a pressure nodal plane in a planar device), although some particles and second-phase liquids will move towards regions of low acoustic velocity, thus providing a mechanism for cell/lipid separation. ${ }^{3}$ Typical devices work in the low-megahertz frequency range with acoustic-pressure amplitudes on the order of hundreds of kilopascals, generating forces on cells of a few, or a few tens, of piconewtons.

There are a number of ways to establish appropriate acoustic fields, including surface-acoustic-wave ${ }^{4}$ and platewave excitation. ${ }^{5}$ The most straightforward excitation technique for use on a microfluidic scale is probably by employing a subwavelength planar resonator. ${ }^{6}$ The majority of our work has

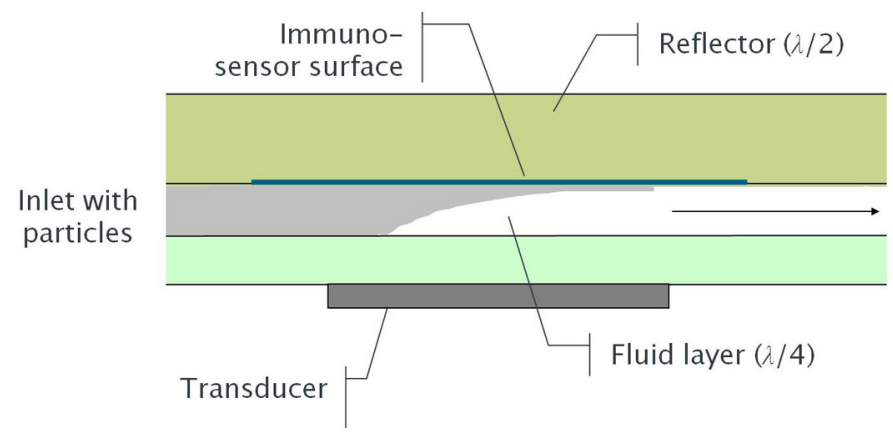

Figure 1. Biosensor enhancement using an acoustic field to force particles (entering from the left) up to a functionalized surface and enhancing cell capture seventyfold. $\lambda$ : Wavelength.
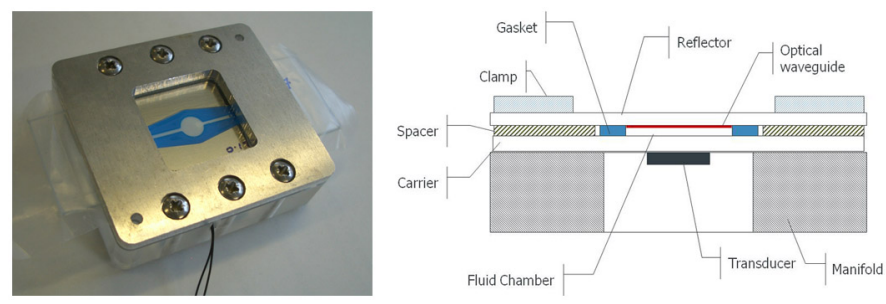

Figure 2. Multimodal manipulator with integrated optical waveguide.

used this approach. We demonstrated acoustic flow-through filtration in etched-silicon and Pyrex ${ }^{\circledR}$ devices, ${ }^{7}$ and developed thick-film printed transducers to drive such devices efficiently. ${ }^{8}$ An interesting possibility offered by 'quarter-wavelength' planar devices (see Figure 1) is the potential to force particles up to a surface in a flow-through biosensor, hence overcoming the diffusion limit on cell capture. ${ }^{9}$ In a collaboration with the University of Cardiff (UK), which improved spore capture on a functionalized surface seventyfold, we modeled both acoustic behavior and spore movement within the field and demonstrated the sensitivity of our approach to small changes in geometric parameters. ${ }^{10}$ 


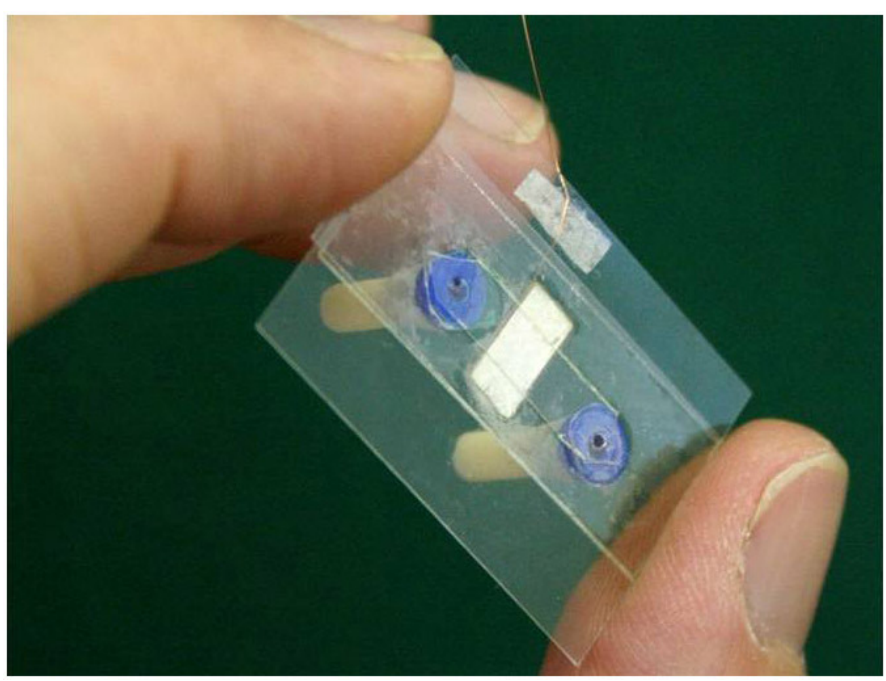

Figure 3. Polymer 'thin-reflector' device.

More recent developments have used different resonant modes within a planar resonator (see Figure 2) to force particles either up to or away from a surface. ${ }^{11}$ We used this to enhance interactions between a functionalized glass surface and polystyrene microbeads and to identify those that bind to the surface by illuminating bound beads using an optical evanescent field. This approach also provides a means of removing nonspecifically-bound beads from the biosensor and has potential for improving DNA and protein assays in terms of detection speed and multiplexing.

A drawback of planar resonators is that they typically lack flexibility and are designed a priori to move particles to a specific position. We have demonstrated that, by switching between two resonant modes, particles can be moved to arbitrary planes between the nodal planes of both modes. ${ }^{12}$ A key aim of our work is to facilitate ultrasonic manipulation within low-cost devices and in multiwell plates. To this end, we have shown that using thin layers (in a structure designed for excitation at the fundamental thickness mode of the entire device) overcomes some of the problematic lateral-force variations affecting quarter-wave devices. ${ }^{13}$ The thin layers used in the structure (see Figure 3 ) allow use of polymers without significant loss of acoustic efficiency. We recently worked with Prokyma Technology Ltd. (UK) to develop polymer resonators that combine ultrasonic and magnetic forces to enhance a bead-based assay for tuberculosis bacteria. This approach enhances the agglomeration speed and improves both the washing efficiency and elution of the beads compared with purely magnetic trapping.

Ultrasonic forces provide a means of manipulating cells without damaging their viability. They work on length scales that fit well with microfluidic applications, but are complementary to optical, dielectrophoretic, and magnetic trapping. Our future priorities are to combine ultrasound with other manipulation techniques, to develop 2D and 3D manipulation strategies, and to investigate tissue-engineering applications.

We are collaborating with Bristol, Dundee, and Glasgow Universities (UK) in the Engineering and Physical Sciences Research Council-funded 'Sonotweezers' project.

\section{Author Information}

\author{
Martyn Hill, Peter Glynne-Jones, and Rosemary J. Boltryk \\ School of Engineering Sciences \\ University of Southampton \\ Southampton, UK
}

Martyn Hill is professor of electromechanical systems. Much of his work is multidisciplinary, including developing the use of ultrasonic radiation forces in microsystems for biological applications and microcharacterization of surface form.

Peter Glynne-Jones graduated from the School of Electronics and Computer Science at the University of Southampton, and was an Institution of Engineering and Technology (IET) scholar. His $2001 \mathrm{PhD}$, Vibration-powered generators for self-powered microsystems, led to the spin-off company Perpetuum. He currently works on manipulation of particles in microsystems using ultrasonic radiation forces.

Rosemary Boltryk is a lecturer and a chartered engineer. She completed a PhD at the University of Southampton in acoustic particle manipulation and maintains an interest in its biomedical applications and aspects of microfluidic design.

\section{Nicholas R. Harris}

School of Electronics and Computer Science

University of Southampton

Southampton, UK

Nick Harris is a senior lecturer. He has research interests in energy harvesting, wireless-sensor networks, and ultrasonics. He has over 100 publications in these and other fields, as well as several patents. He is a member of the IET and a chartered engineer. 


\section{References}

1. J. Hultström, O. Manneberg, K. Dopf, H. M. Hertz, H. Brismar, and M. Wiklund, Proliferation and viability of adherent cells manipulated by standing-wave ultrasound in a microfluidic chip, Ultrasound Med. Biol. 33, pp. 175-181, 2006. doi:10.1016/j.ultrasmedbio.2006.07.024

2. H. Bruus, Theoretical Microfluidics, Oxford Univ. Press, New York, 2008.

3. F. Petersson, A. Nilsson, C. Holm, H. Jonsson, and T. Laurell, Continuous separation of lipid particles from erythrocytes by means of laminar flow and acoustic standing wave forces, Lab on a Chip 5 (1), pp. 20-22, 2005. doi:10.1039/b405748c

4. L. Y. Yeo and J. R. Friend, Ultrafast microfluidics using surface acoustic waves, Biomicrofluid. 3 (1), p. 012002, 2009. doi:10.1063/1.3056040

5. A. Haake and J. Dual, Contactless micromanipulation of small particles by an ultrasound field excited by a vibrating body, J. Acoust. Soc. Am. 117 (5), pp. 2752-2760, 2005. doi:10.1121/1.1874592

6. J. J. Hawkes and W. T. Coakley, Force field particle filter, combining ultrasound standing waves and laminar flow, Sens. Act. B 75 (3), pp. 213-222, 2001. doi:10.1016/S09254005(01)00553-6

7. N. R. Harris, M. Hill, S. P. Beeby, Y. Shen, N. M. White, J. J. Hawkes, and W. T. Coakley, A silicon microfluidic ultrasonic separator, Sens. Act. B 95 (1-3), pp. 425-434, 2003. doi:10.1016/S0925-4005(03)00448-9

8. N. R. Harris, M. Hill, R. N. Torah, R. J. Townsend, S. P. Beeby, N. M. White, and J. Ding, A multilayer thick-film PZT actuator for MEMS applications, Sens. Act. A 132, pp. 311-316, 2006. doi:10.1016/j.sna.2006.06.006

9. M. Hill, The selection of layer thicknesses to control acoustic radiation force profiles in layered resonators, J. Acoust. Soc. Am. 114 (5), pp. 2654-2661, 2003. doi:10.1121/1.1616581

10. S. P. Martin, R. J. Townsend, L. A. Kuznetsova, K. A. J. Borthwick, M. Hill, M. B. McDonnell, and W. T. Coakley, Spore and micro-particle capture on an immunosensor surface in an ultrasound standing wave system, Biosens. Bioelectron. 21 (5), pp. 758-767, 2005. doi:10.1016/j.bios.2005.01.013

11. P. Glynne-Jones, R. J. Boltryk, M. Hill, F. Zhang, L. Dong, J. S. Wilkinson, T. Brown, T. Melvin, and N. R. Harris, Multi-modal particle manipulator to enhance bead-based bioassays, Ultrasonics 50 (2), pp. 235-239, 2010. doi:10.1016/j.ultras.2009.09.025

12. P. Glynne-Jones, R. J. Boltryk, N. R. Harris, A. W. J. Cranny, and M. Hill, Mode-switching: a new technique for electronically varying the agglomeration position in an acoustic particle manipulator, Ultrasonics 50, pp. 68-75, 2010. doi:10.1016/j.ultras.2009.07.010

13. P. Glynne-Jones, R. J. Boltryk, M. Hill, N. R. Harris, and P. Baclet, Robust acoustic particle manipulation: a thin-reflector design for moving particles to a surface, J. Acoust. Soc. Am. 126 (3), pp. EL75-EL79, 2009. doi:10.1121/1.3186800 